\title{
HYPERBOLIC METRICS ON FINITE-DIMENSIONAL TEICHMÜLLER SPACES
}

\author{
S.L. Krushkal'
}

\section{Introduction. Statement of result}

Teichmüller spaces are important in different fields and also interesting as model examples in the general theory of complex manifolds, especially hyperbolic ones. Thus it is of great interest to establish how the general problems deriving from the theory of complex manifolds are solved for these spaces. One such problem is the question of the coincidence of invariant hyperbolic Carathéodory and Kobayashi metrics; it was posed in [1], [4]. This question proves to be very important also for the variational problems of geometric function theory (see [9]).

For the finite-dimensional Teichmüller spaces $T(g, n)$, which correspond to Riemann surfaces of finite conformal type $(g, n)$ with $2 g+n>2$, the situation is as follows. There is an important theorem by Royden [12], asserting that on these spaces the Kobayashi metric coincides with their intrinsic Teichmüller metric, which is defined by using quasiconformal mappings; later Gardiner [13] has extended this result also to the infinite-dimensional Teichmüller spaces.

On the other hand, it was established by the author in [7], [8] that, on the Teichmüller spaces $T(g, n)$ of a dimension greater than two, the Carathéodory metric on the whole is smaller than the Teichmüller-Kobayashi metric, so the problem has a negative solution. The arguments used there assume the existence on the surfaces of three linear independent holomorphic quadratic differentials (with fully determined properties), and thus they are suited for $\operatorname{dim} T(g, n) \geq 3$ only. The question remained open for the Teichmüller spaces of dimension 2 ; there are the spaces $T(0,5)$ of the spheres with five punctures and $T(1,2)$ of the tori with two punctures, which are biholomorphically equivalent.

The goal of the present paper is to show that the metrics do not coincide for these spaces either. The reasons proposed below are valid simultaneously for all spaces $T(g, n)$ of $\operatorname{dim}>1$ with punctures; for $\operatorname{dim} T(g, n) \geq 3$ that gives a new (and more effective) proof that the above metrics do not coincide.

On account of applications, it is important to have sufficient conditions on holomorphic disks in $T(g, n)$ that provide the coincidence of invariant metrics on these disks. Kra [6] has shown this to be true for the Abelian Teichmüller disks defined by quadratic differentials with zeros of even order. An analogous result is established in [10] for the universal Teichmüller space; it can be extended also to the Teichmüller spaces of finitely many punctured disks. 
Here we prove

Theorem. Let $\operatorname{dim} T(g, n+1)=3 g-2+n>1(g \geq 0, n \geq 0)$ and $\pi: T(g, n+1) \rightarrow T(g, n)$ be the canonical fiber space in which the projection is induced by forgetting a puncture on a base surface of type $(g, n+1)$. Then, in fibers $\pi^{-1}(x)$, the hyperbolic Carathéodory and Teichmüller-Kobayashi metrics (on the space $T(g, n+1))$ do not coincide.

\section{Some auxiliary constructions and results}

First of all, we recall that if $M$ is a complex manifold (finite-dimensional or even Banach), its Carathéodory metric is

$$
c_{M}(x, y)=\sup \{\varrho(h(x), h(y)): h \in \operatorname{Hol}(M, \Delta)\}
$$

where $\varrho$ is the hyperbolic metric in the unit disk $\Delta=\{z:|z|<1\}$ of curvature -4 , i.e., with the differential element $d \varrho=\left(1-|z|^{2}\right)^{-1}$, and the Kobayashi metric $d_{M}(x, y)$ is the greatest of all pseudometrics $d(\cdot, \cdot)$ on $M$ satisfying the inequality

$$
d\left(h\left(z^{\prime}\right), h\left(z^{\prime \prime}\right)\right) \leq \varrho\left(z^{\prime}, z^{\prime \prime}\right), \quad h \in \operatorname{Hol}(\Delta, M) .
$$

Let $X_{0}$ be a given Riemann surface of conformal type $(g, n+1)$, i.e, of genus $g$ with $n+1$ punctures, where $2 g-2+n>0$. Fixing a conformal structure on $X_{0}$ we consider this surface as the initial point in $T(g, n+1)$. Let $p_{0}$ be a puncture on $X_{0}$ and

$$
\tilde{X}_{0}=X_{0} \cup\left\{p_{0}\right\}
$$

We uniformize the surfaces $X_{0}$ and $\tilde{X}_{0}$ by finitely generated Fuchsian groups $\Gamma$ and $\tilde{\Gamma}$ of the first kind and without torsion, acting discontinuously on upper and lower half-planes

$$
U=\{z: \operatorname{Im} z>0\}, \quad U^{*}=\{z: \operatorname{Im} z<0\}
$$

Then, as well known,

$$
T(g, n+1) \cong T(\Gamma)=L_{\infty}(U, \Gamma)_{1} /\left\{\mu \in L_{\infty}(U, \Gamma)_{1}:\left.w^{\mu}\right|_{R}=\mathrm{id}\right\}
$$

where

$$
L_{\infty}(U, \Gamma)_{1}=\left\{\mu \in L_{\infty}(\mathbf{C}): \mu \mid U^{*}=0, \quad(\mu \circ \gamma) \bar{\gamma}^{\prime} / \gamma^{\prime}=\mu, \quad \gamma \in \Gamma ;\|\mu\|_{\infty}<1\right\}
$$

and $w^{\mu}$ is the quasiconformal automorphism of $\mathbf{C}$ with the Beltrami coefficient $\mu \in L_{\infty}(U, \Gamma)_{1}$ and fixed points $0,1, \infty$. 
The conformality of $w^{\mu}$ in $U^{*}$ allows us to consider the mapping

$$
\Phi: \mu \rightarrow\left\{w^{\mu}, z\right\}=\frac{\left(w^{\mu}\right)^{\prime \prime \prime}}{\left(w^{\mu}\right)^{\prime}}-\frac{3}{2}\left[\frac{\left(w^{\mu}\right)^{\prime \prime}}{\left(w^{\mu}\right)^{\prime}}\right]^{2}, \quad z \in U^{*},
$$

which correctly defines a biholomorphic isomorphism of the space $T(g, n+1)$ onto a bounded domain in the space $B_{2}\left(U^{*}, \Gamma\right)$ of holomorphic solutions of the equation $(\psi \circ \gamma) \gamma^{\prime 2}=\psi, \gamma \in \Gamma$, in $U^{*}$, with the norm $\|\psi\|=\sup _{U^{*}}(\operatorname{Im} z)^{2}|\psi(z)|$. Here, $\Phi$ itself acts holomorphically from $L_{\infty}(U, \Gamma)_{1}$ to $B_{2}\left(U^{*}, \Gamma\right)$ and, in particular,

$$
d \Phi(0) \mu=-\frac{6}{\pi} \iint_{U} \frac{\mu(\zeta) d \xi d \eta}{\zeta-z)^{4}} \quad\left(\zeta=\xi+i \eta, z \in U^{*}\right) .
$$

We identify $T(g, n+1)$ with its embedding in $B_{2}\left(U^{*}, \Gamma\right)$. An analogous construction is valid, of course, also for $T(g, n) \cong T(\tilde{\Gamma})$; the corresponding projection for this space we will denote by $\tilde{\Phi}$.

The Teichmüller metric on $T(g, n+1)$ is

$$
\tau_{\Gamma}(\Phi(\mu), \Phi(\nu))=\frac{1}{2} \inf \left\{\log K\left(w^{\mu^{\prime}} \circ\left(w^{\nu^{\prime}}\right)^{-1}: \Phi\left(\mu^{\prime}\right)=\Phi(\mu), \Phi\left(\nu^{\prime}\right)=\Phi(\nu)\right\},\right.
$$

where $K\left(w^{\sigma}\right)=\left(1+\|\sigma\|_{\infty}\right) /\left(1-\|\sigma\|_{\infty}\right)$.

One could easily establish that

$$
c_{T}(x, y) \leq d_{T}(x, y) \leq \tau_{T}(x, y) .
$$

The identical embedding $j: X_{0} \hookrightarrow \tilde{X}_{0}$ "forgetting a puncture" induces an isometrical isomorphism $j_{*}(\mu): L_{\infty}(U, \Gamma) \rightarrow L_{\infty}(U, \tilde{\Gamma})$ by

$$
j_{*}(\mu) \circ J=\mu J^{\prime} / \bar{J}^{\prime}
$$

where $J: U \rightarrow U$ is a lifting of $j$ from $x_{0}$ onto $U$; this isomorphism is compatible with the projections $\Phi$ and $\tilde{\Phi}$. Thus $j$ determines a holomorphic fiber space

$$
\pi: T(g, n+1) \rightarrow T(g, n),
$$

which is mentioned in the theorem (this fibering is a holomorphic disk family admitting only a real local $C^{\infty}$-trivialization).

With this fibering is connected another fiber space

$$
\pi_{0}: F(g, n) \rightarrow T(g, n),
$$

which was introduced by Bers. Here

$$
F(g, n) \cong F(\tilde{\Gamma})=\left\{(\tilde{\Phi}(\mu), z) \in T(\tilde{\Gamma}) \times \mathbf{C}: \mu \in L_{\infty}(U, \tilde{\Gamma})_{1}, z \in w^{\mu}(U)\right\},
$$


with the projection $\pi_{0}:(\tilde{\Phi}(\mu), z) \mapsto \tilde{\Phi}(\mu)$.

According to Bers' isomorphism theorem [2], the fiber spaces (3) and (4) are isomorphic; thus there exists a biholomorphic isomorphism

$$
T(g, n+1) \cong F(g, n)
$$

compatible with the projections. It is defined by the map

$$
\mu \mapsto\left(\tilde{\Phi}\left(j_{*}(\mu)\right), w^{j_{*}(\mu)}\left(z_{0}\right)\right),
$$

where $z_{0}$ is a fixed preimage of the point $p_{0}$ in $U$ by a universal holomorphic covering $U \rightarrow \tilde{X}_{0}$.

The following result of Kra [6] and Nag [11], which we essentially will use, is established with the aid of the isomorphism theorem.

Proposition (Kra-Nag). The fibers $\pi^{-1}(x)$ (for $(g, n) \neq(0,3)$ ) are not totally geodesic in $T(g, n+1)$ relative to the metric $\tau_{T}$, i.e., they are not $T e-$ ichmüller disks, and $\tau_{T}$ is connected with the hyperbolic metric hyp on the fiber $\pi^{-1}(x)$ (which is isometrically pull-backed from $\Delta$ by a holomorphic embedding $\left.\Delta \rightarrow \pi^{-1}(x)\right)$ by the strong inequality

$$
\tau_{T}\left(z_{1}, z_{2}\right)<\operatorname{hyp}\left(z_{1}, z_{2}\right) \quad\left(z_{1}, z_{2} \in \pi^{-1}(x)\right) .
$$

Recall also that the Teichmüller disks $\Delta_{\varphi}$ in $T(g, n+1)$ have the form

$$
\Delta_{\varphi}=\{\Phi(t \bar{\varphi} /|\varphi|): t \in \Delta\}
$$

where $\varphi$ is a holomorphic quadratic differential on $X_{0}$. The space of such differentials (with $L_{1}$-norm) will be denoted by $Q\left(x_{0}\right)$ or $Q(\Gamma)$; that is the cotangent space of $T(g, n+1)$ at the point $X_{0}$.

\section{Proof of the theorem}

Let us now turn to the proof of the theorem. We model $T(g, n+1)$ and $T(g, n)$ again as $T(\Gamma)$ and $T(\tilde{\Gamma})$, and take their embeddings in $B_{2}\left(U^{*}, \Gamma\right)$ (note that $B_{2}\left(U^{*}, \tilde{\Gamma}\right)$ is isometrically embedded into $B_{2}\left(U^{*}, \Gamma\right)$ by $\left.\varphi \mapsto(\varphi \circ J) J^{\prime 2}\right)$.

It is sufficient to establish the validity of the assertion of the theorem for the initial fiber $\pi^{-1}(0)$ only. The case of a general fiber $\pi^{-1}(\varphi), \varphi \in T(\tilde{\Gamma})$ (and an analogous case of an arbitrary point from $\pi^{-1}(0) \backslash\{0\}$ ) can be reduced to such ones by passing from a quasifuchsian group $\Gamma_{\mu}=w^{\mu} \Gamma\left(w^{\mu}\right)^{-1}$ to its Fuchsian equivalent using a conformal mapping of the domain $w^{\mu}(U)$ onto $U$; this leads us to the so-called admissible bijection of $T(\Gamma)$, which preserves the metrics. 
We will prove that for the point $\varphi=0 \in T(\Gamma)$ (or, equivalently, for the basepoint $x_{0}$, to which the point $\left(0, z_{0}\right) \in F(\tilde{\Gamma})$ corresponds) there must exist a neighbourhood in the fiber $\pi^{-1}(0)$ over $0 \in T(\tilde{\Gamma})$, in which the equality

$$
c_{T}(\psi, 0)<\tau_{T}(\psi, 0)
$$

holds.

Assuming the contrary, we find a sequence of Schwarzians $\left\{\psi_{m}\right\} \subset \pi^{-1}(0)$ which converges to 0 in $B_{2}\left(U^{*}, \Gamma\right)$, and that sequence corresponds on $X_{0}$ to a sequence of points $\left\{p_{m}\right\}$, converging to $p_{0}$ (for example, in a hyperbolic metric on $\left.\tilde{X}_{0}\right)$ such that $\psi_{m}$ represents in $T(\Gamma)$ the marked Riemann surface

$$
X_{m}=\tilde{X}_{0} \backslash\left\{p_{m}\right\}
$$

(with puncture $p_{m}$ on $\tilde{X}_{0}$ instead of $p_{0}$ ) and

$$
c_{T}\left(\psi_{m}, 0\right)=\tau_{T}\left(\psi_{m}, 0\right), \quad m=1,2, \ldots
$$

Let

$$
\left\{h_{m}\right\} \subset \operatorname{Hol}(T(\Gamma), \Delta), \quad h_{m}(0)=0,
$$

be the corresponding sequence of holomorphic functions on which the distances $c_{T}\left(\psi_{m}, 0\right)$ are attained, i.e.,

$$
\varrho\left(h_{m}\left(\psi_{m}\right), 0\right)=c_{T}\left(\psi_{m}, 0\right),
$$

and suppose that $k_{m} \mu_{m}$ are extremal Beltrami differentials with

$$
\mu_{m}=\frac{\bar{\varphi}_{m}}{\left|\varphi_{m}\right|}, \quad\left(0<\kappa_{m}<1, \varphi_{m} \in Q(\Gamma) \backslash\{0\}\right)
$$

on which the Teichmüller distance $\tau_{T}\left(\psi_{m}, 0\right)$ is realized (i.e.,

$$
\tau_{T}\left(\psi_{m}, 0\right)=\frac{1}{2} \log \left[\left(1+k_{m}\right) /\left(1-k_{m}\right)\right],
$$

$m=1,2, \ldots)$. We normalize the quadratic differentials $\varphi_{m}$ so that

$$
\left\|\varphi_{m}\right\|_{L_{1}}=1 \text { for all } m=1,2, \ldots
$$

Consider now the corresponding Teichmüller disks

$$
\Delta_{\varphi_{m}}=\left\{\Phi\left(t \frac{\bar{\varphi}_{m}}{\left|\varphi_{m}\right|}\right): t \in \Delta\right\} \subset T(\Gamma) .
$$


One can also assume the functions $h_{m}$ to be taken such that

$$
h_{m} \circ \Phi\left(t \frac{\bar{\varphi}_{m}}{\left|\varphi_{m}\right|}\right)=t \quad(t \in \Delta)
$$

and hence

$$
d\left(h_{m} \circ \Phi\right)(0) t \mu_{m}=t \quad(t \in \mathbf{C}) .
$$

Turning, if necessary, to the subsequences, we may assume that $\varphi_{m}$ converges in $Q(\Gamma)$ (and locally uniformly in $\Delta$ ) to $\varphi_{0} \in Q(\Gamma) \backslash\{0\}$ with $\left\|\varphi_{0}\right\|=1$, and $h_{m}$ converge locally uniformly in $T(\Gamma)$ to a function $h_{0} \in \operatorname{Hol}(T(\Gamma), \Delta) \backslash\{0\}$. Besides, the disks $\Delta-\varphi_{m}$ converge to $\Delta_{\varphi_{0}}$ in a Teichmüller metric locally, and by virtue of $(8)$

$$
d\left(h_{0} \circ \Phi\right)(0) t \mu_{0}=t \quad\left(\mu_{0}=\bar{\varphi}_{0} /\left|\varphi_{0}\right|, t \in \mathbf{C}\right),
$$

which means $d h_{0}(0)$ is an isometry on the tangent line $T_{0} \Delta_{\varphi_{0}}$ to $\Delta_{\varphi_{0}}$ in 0 .

Let us also show that this tangent cannot be transversal to the tangent line $T_{0} \pi^{-1}(0)$, i.e., that $T_{0} \Delta_{\varphi_{0}}$ and $T_{0} \pi^{-1}(0)$ must coincide. Indeed, if the equation for the fiber $\pi^{-1}(0)$ in $B_{2}\left(U^{*}, \Gamma\right)$ is

$$
\psi=f_{0}(t)=\sum_{m=1}^{\infty} t^{m} f_{0}^{(m)}(0) / m ! \quad\left(f_{0}(t) \equiv f(z, t) \in \operatorname{Hol}(\Delta, T(\Gamma))\right.
$$

then, for small $|t|$, we have

$$
\psi=t f_{0}^{\prime}(0)+O\left(t^{2}\right) \quad\left(f_{0}^{\prime}(0) \neq 0\right)
$$

and so $T_{0} \pi^{-1}(0)=\left\{t f_{0}^{\prime}(0): t \in \mathbf{C}\right\}$; we assume below that $f_{0}$ is the abovementioned biholomorphic embedding. It follows from (1) that

$$
f_{0}(z, t)=-\frac{6}{\pi} \iint_{U} \frac{\mu(\xi) d \xi d \eta}{(\xi-z)^{4}}+O\left(\|\mu\|^{2}\right)
$$

for any $\mu \in L_{\infty}(U, \Gamma)_{1}$ with $\Phi(\mu)=f_{0}(t)$. In particular, due to the well-known Ahlfors-Weill theorem on quasiconformal extension, one can take

$$
\mu(\zeta)=-2 \eta^{2} f_{0}(\bar{\xi}, t)
$$

(and then $\left\{w^{\mu}, z\right\}=f_{0}(z, t)$ ). 
Let us return to our $\psi_{m}$. Let these $\psi_{m}$ correspond to $t=t_{m}$ from $\Delta$, i.e., $\psi_{m}=f_{0}\left(t_{m}\right)$. Then we have from (11)

$$
\frac{12}{\pi} \iint_{U} \frac{\eta^{2} f_{0}\left(\bar{\zeta}, t_{m}\right)}{(\zeta-z)^{4}} d \xi d \eta+O\left(t_{m}^{2}\right)=-\frac{6 k_{m}}{\pi} \iint_{U} \frac{\mu_{m}(\zeta) d \xi d \eta}{(\zeta-z)^{4}}+O\left(k_{m}^{2}\right)
$$

(where $k_{m}<\left|t_{m}\right|$ ) or, rememberring (10),

$$
\frac{12}{\pi} \iint_{U} \frac{\eta^{2} \bar{f}_{0}^{\prime}(0)}{(\zeta-z)^{4}} d \xi d \eta=-\frac{6}{\pi} \frac{k_{m}}{t_{m}} \iint_{U} \frac{\mu_{m} d \xi d \eta}{(\zeta-z)^{4}}+O\left(t_{m}\right)+O\left(\frac{k_{m}^{2}}{t_{m}}\right) .
$$

By applying the Lebesgue theorem on majorized convergence to the integral on the right-hand side and taking into account that the left-hand integral is equal to $f_{0}^{\prime}(0)$, we obtain by the well-known reproduction formula for the elements from $B_{2}\left(U^{*}, \Gamma\right)$

$$
f_{0}^{\prime}(0)=-\frac{6 a}{\pi} \int_{U} \int \frac{\left(\bar{\varphi}_{0} /\left|\varphi_{0}\right|\right) d \xi d \eta}{(\zeta-z)^{4}},
$$

where $a=\lim _{m \rightarrow \infty}\left(k_{m} / t_{m}\right) \neq 0$ (because of $\varphi_{0} \neq 0$ and $f_{0}^{\prime}(0) \neq 0$ ); but that means the coincidence of directions for tangent lines of $\pi^{-1}(0)$ and of $\Delta_{\varphi_{0}}$ in 0 .

Now the proof of the theorem is completed in the following way. For the function

$$
h_{0} \circ f_{0}: \Delta \rightarrow \pi^{-1}(0) \rightarrow \Delta
$$

we have, by virtue of (9)

$$
\left|d\left(h_{0} \circ f_{0}\right)(0) t\right|=\left|d h_{0}(0) d f_{0}(0) t\right|=|t| .
$$

Hence, due to Schwarz' lemma, the equality $h_{0} \circ f_{0}(t)=e^{i \alpha} t, \alpha \in \mathbf{R}$, must hold for all $t \in \Delta$, or, equivalently, in the whole fiber $\pi^{-1}(0)$ we have

$$
c_{T}(\psi, 0)=\operatorname{hyp}(\psi, 0) .
$$

Comparing that with (2), we see that in $\pi^{-1}(0)$

$$
\tau_{T}(\psi, 0) \geq \operatorname{hyp}(\psi, 0)
$$

must hold, contrary to (5), (in fact, by virtue of the coincidence of $\tau_{T}$ with $d_{T}$, here can only the equality be, which means that the fiber $\pi^{-1}(0)$ is Teichmüllerian).

The contradiction thus obtained proves that for all $\psi$, closed to 0 in $\pi^{-1}(0)$, the strong inequality (6) should be valid. Thus the theorem is proved.

I am grateful to the referee for his helpful remarks. 


\section{References}

[1] A crash course on Kleinian groups: edited by L. Bers and I. Kra. - Lecture Notes in Mathematics 400. Springer-Verlag, Berlin-Heidelberg-New York, 1974.

[2] Bers, L.: Fiber spaces over Teichmüller spaces. - Acta Math. 130, 1973, 89-126.

[3] Gardiner, F.P.: Approximation of infinite dimensional Teichmüller spaces. - Trans. Amer. Math. Soc. 282, 1984, 367-383.

[4] Kobayashi, S.: Intrinsic distances, measures and geometric function theory. - Bull. Amer. Math. Soc. 82, 1976, 357-416.

[5] KrA, I.: On the Nielsen-Thurston-Bers type of some selfmaps of Riemann surfaces. - Acta Math. 146, 1981, 231-270.

[6] Kra, I.: The Carathéodory metric on Abelian Teichmüller disks. - J. Analyse Math. 40, 1981, 129-143.

[7] KRUshkaL', S.L.: Invariant metrics in Teichmüller spaces. - Siberian Math. J. 22:2, 1981, 209-212; 29:1, 1988, 219.

[8] KRUShKAL', S.L.: Invariant metrics on the spaces of closed Riemann surfaces. - Siberian Math. J. 26:2, 1985, 108-114; 29:1, 1988, 219.

[9] Krushkal', S.L.: A new method for solving of variational problems in the theory of quasiconformal mappings. - Siberian Math. J. 29:2, 1988, 105-114.

[10] KrushKal', S.L.: Grunsky coefficient inequalities, Carathéodory metric and extremal quasiconformal mappings. - Comment. Math. Helv. 64, 1989, 650-660.

[11] NAG, S.: Non-geodesic discs embedded in Teichmüller spaces. - Amer. J. Math. 104, 1982, 399-408.

[12] Royden, H.L.: Automorphisms and isometries of Teichmüller spaces. - Advances in the theory of Riemann surfaces. Ann. of Math. Stud. 66, Princeton University Press, Princeton, N.J., 1971, 369-383.

Siberian Branch of the USSR Academy of Sciences Institute of Mathematics

Novosibirsk, 630090

U.S.S.R.

Received 31 May 1989 